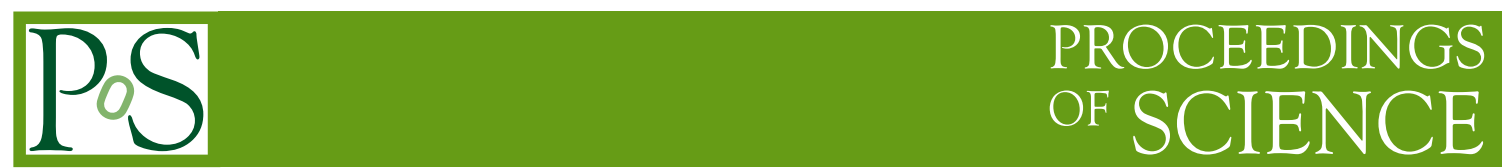

\title{
Results from the CUORE experiment
}

L. Marini ${ }^{* 1,17}$, D. Q. Adams ${ }^{2}$, C. Alduino ${ }^{2}$, K. Alfonso ${ }^{3}$, F. T. Avignone III $^{2}$, O. Azzolini ${ }^{4}$, G. Bari ${ }^{5}$, F. Bellini ${ }^{6,7}$, G. Benato ${ }^{1}$, A. Bersani ${ }^{8}$, M. Biassoni ${ }^{9}$, A. Branca ${ }^{10,11}$, C. Brofferio ${ }^{12,9}$, C. Bucci ${ }^{13}$, A. Caminata ${ }^{8}$, A. Campani ${ }^{14,8}$, L. Canonica ${ }^{15,13}$, X. G. Cao ${ }^{16}$, S. Capelli ${ }^{12,9}$, L. Cappelli ${ }^{13,1,17}$, L. Cardani ${ }^{7}$, P. Carniti ${ }^{12,9}$, N. Casali ${ }^{7}$, L. Cassina ${ }^{12,9}$, D. Chiesa ${ }^{12,9}$, N. Chott $^{1}$, M. Clemenza ${ }^{12,9}$, S. Copello ${ }^{18,13}$, C. Cosmelli6,7, O. Cremonesi ${ }^{9}$, R. J. Creswick$^{2}$, J. S. Cushman ${ }^{19}$, A. D'Addabbo ${ }^{13}$, D. D'Aguanno ${ }^{13,20}$, I. Dafinei ${ }^{7}$, C. J. Davis ${ }^{19}$, S. Dell'Oro ${ }^{21}$, M. M. Deninno5 , S. Di Domizio ${ }^{14,8}$, V. Dompè ${ }^{13,18}$, A. Drobizhev ${ }^{1,17}$, D. Q. Fang ${ }^{16}$, M. Faverzani ${ }^{12,9}$, E. Ferri ${ }^{12,9}$, F. Ferroni ${ }^{6,7}$, E. Fiorini ${ }^{9,12}$, M. A. Franceschi ${ }^{22}$, S. J. Freedman ${ }^{17,1, a}$, B. K. Fujikawa ${ }^{17}$, A. Giachero ${ }^{12,9}$, L. Gironi ${ }^{12,9}$, A. Giuliani ${ }^{23}$, L. Gladstone ${ }^{15}$, P. Gorla ${ }^{13}$, C. Gotti ${ }^{12,9}$, T. D. Gutierrez ${ }^{24}$, K. Han ${ }^{25}$, K. M. Heeger ${ }^{19}$, R. Hennings-Yeomans ${ }^{1,17}$, R. G. Huang ${ }^{1}$, H. Z. Huang ${ }^{3}$, J. Johnston ${ }^{15}$, G. Keppel ${ }^{4}$, Yu. G. Kolomensky ${ }^{1,17}$, A. Leder ${ }^{15}$, C. Ligi ${ }^{22}$, Y. G. Ma ${ }^{16}$, M. Martinez ${ }^{6,7,26}$, R. H. Maruyama ${ }^{19}$, Y. Mei ${ }^{17}$, N. Moggi ${ }^{27,5}$, S. Morganti ${ }^{7}$, S. S. Nagorny ${ }^{13,18}$, T. Napolitano ${ }^{22}$, M. Nastasi ${ }^{12,9}$, C. Nones ${ }^{28}$, E. B. Norman ${ }^{29,30}$, V. Novati ${ }^{23}$, A. Nucciotti ${ }^{12,9}$, I. Nutini ${ }^{13,18}$, T. O’Donnell ${ }^{21}$, J. L. Ouellet ${ }^{15}$, C. E. Pagliarone ${ }^{13,20}$, M. Pallavicini ${ }^{14,8}$, V. Palmieri ${ }^{4, a}$, L. Pattavina ${ }^{13}$, M. Pavan ${ }^{12,9}$, G. Pessina ${ }^{9}$, C. Pira ${ }^{4}$, S. Pirro ${ }^{13}$, S. Pozzi ${ }^{12,9}$, E. Previtali ${ }^{9}$, A. Puiu ${ }^{12,9}$, F. Reindl ${ }^{7}$, C. Rosenfeld ${ }^{2}$, C. Rusconi ${ }^{2,13}$, M. Sakai $^{3}$, S. Sangiorgio ${ }^{29}$, D. Santone ${ }^{13,31}$, B. Schmidt ${ }^{17}$, N. D. Scielzo ${ }^{29}$, V. Singh ${ }^{1}$, M. Sisti ${ }^{12,9}$, D. Speller ${ }^{19}$, L. Taffarello ${ }^{10}$, F. Terranova ${ }^{12,9}$, C. Tomei ${ }^{7}$, M. Vignati ${ }^{7}$, S. L. Wagaarachchi ${ }^{1,17}$, B. S. Wang ${ }^{29,30}$, H. W. Wang ${ }^{16}$, B. Welliver ${ }^{17}$, J. Wilson ${ }^{2}$, K. Wilson ${ }^{2}$, L. A. Winslow ${ }^{15}$, T. Wise ${ }^{19,32}$, L. Zanotti ${ }^{12,9}$, G. Q. Zhang ${ }^{16}$, S. Zimmermann ${ }^{33}$, and S. Zucchelli ${ }^{27,5}$, on the behalf of the CUORE collaboration 
${ }^{1}$ Department of Physics, University of California, Berkeley, CA 94720, USA

2 Department of Physics and Astronomy, University of South Carolina, Columbia, SC 29208, USA

${ }^{3}$ Department of Physics and Astronomy, University of California, Los Angeles, CA 90095, USA

${ }^{4}$ INFN - Laboratori Nazionali di Legnaro, Legnaro (Padova) I-35020, Italy

${ }^{5}$ INFN - Sezione di Bologna, Bologna I-40127, Italy

${ }^{6}$ Dipartimento di Fisica, Sapienza Università di Roma, Roma I-00185, Italy

${ }^{7}$ INFN - Sezione di Roma, Roma I-00185, Italy

${ }^{8}$ INFN - Sezione di Genova, Genova I-16146, Italy

${ }^{9}$ INFN - Sezione di Milano Bicocca, Milano I-20126, Italy

${ }^{10}$ INFN - Sezione di Padova, Padova I-35131, Italy

${ }^{11}$ Dipartimento di Fisica e Astronomia, Università di Padova, I-35131 Padova, Italy

${ }^{12}$ Dipartimento di Fisica, Università di Milano-Bicocca, Milano I-20126, Italy

${ }^{13}$ INFN - Laboratori Nazionali del Gran Sasso, Assergi (L'Aquila) I-67100, Italy

${ }^{14}$ Dipartimento di Fisica, Università di Genova, Genova I-16146, Italy

${ }^{15}$ Massachusetts Institute of Technology, Cambridge, MA 02139, USA

${ }^{16}$ Shanghai Institute of Applied Physics, Chinese Academy of Sciences, Shanghai 201800, China

${ }^{17}$ Nuclear Science Division, Lawrence Berkeley National Laboratory, Berkeley, CA 94720, USA

${ }^{18}$ INFN - Gran Sasso Science Institute, L'Aquila I-67100, Italy

${ }^{19}$ Wright Laboratory, Department of Physics, Yale University, New Haven, CT 06520, USA

${ }^{20}$ Dipartimento di Ingegneria Civile e Meccanica, Università degli Studi di Cassino e del Lazio Meridionale, Cassino I-03043, Italy

${ }^{21}$ Center for Neutrino Physics, Virginia Polytechnic Institute and State University, Blacksburg, Virginia 24061, USA

${ }^{22}$ INFN - Laboratori Nazionali di Frascati, Frascati (Roma) I-00044, Italy

${ }^{23}$ CSNSM, Univ. Paris-Sud, CNRS/IN2P3, UniversitÃČĈAl' Paris-Saclay, 91405 Orsay, France

${ }^{24}$ Physics Department, California Polytechnic State University, San Luis Obispo, CA 93407, USA

${ }^{25}$ INPAC and School of Physics and Astronomy, Shanghai Jiao Tong University; Shanghai Laboratory for Particle Physics and Cosmology, Shanghai 200240, China

${ }^{26}$ Laboratorio de Fisica Nuclear y Astroparticulas, Universidad de Zaragoza, Zaragoza 50009, Spain

${ }^{27}$ Dipartimento di Fisica e Astronomia, Alma Mater Studiorum - Università di Bologna, Bologna I-40127, Italy

${ }^{28}$ Service de Physique des Particules, CEA / Saclay, 91191 Gif-sur-Yvette, France

${ }^{29}$ Lawrence Livermore National Laboratory, Livermore, CA 94550, USA

${ }^{30}$ Department of Nuclear Engineering, University of California, Berkeley, CA 94720, USA

${ }^{31}$ Dipartimento di Scienze Fisiche e Chimiche, Università dell'Aquila, L'Aquila I-67100, Italy

${ }^{32}$ Department of Physics, University of Wisconsin, Madison, WI 53706, USA

${ }^{33}$ Engineering Division, Lawrence Berkeley National Laboratory, Berkeley, CA 94720, USA

${ }^{a}$ Deceased

E-mail: lmarini@berkeley.edu 
The Cryogenic Underground Observatory for Rare Events (CUORE) is the first bolometric experiment operated at about $10 \mathrm{mK}$ reaching the ton-scale. CUORE main goal is the search for neutrinoless double beta decay in ${ }^{130} \mathrm{Te}$, while it is suitable also for other kinds of rare event searches, such as dark matter and axions. The detector is situated at Laboratori Nazionali del Gran Sasso (LNGS), Italy, and consists in an array of $988 \mathrm{TeO}_{2}$ crystals, being both the detector and the source of the decay. Since the beginning of 2017, CUORE operations had both phases for physics data taking and campaigns of detector optimization to obtain the maximum from its potential. Here we briefly discuss one of the procedures for the optimization of the detector regarding noise reduction and report the achievements of CUORE concerning energy resolution and background in the region of interest after the first few months of data taking. Finally we conclude by examining the results from our first neutrinoless double beta decay analysis published at the beginning of 2018, which with a total $\mathrm{TeO}_{2}$ exposure of $86.3 \mathrm{~kg} \cdot \mathrm{y}$ allowed to place a lower limit on the decay half-life of $T_{1 / 2}^{0 v}\left({ }^{130} \mathrm{Te}\right)>1.3 \times 10^{25} \mathrm{yr}(90 \%$ C.L.) .

XIV International Conference on Heavy Quarks and Leptons (HQL2018)

May 27- June 1, 2018

Yamagata Terrsa, Yamagata,Japan

* Speaker. 


\section{1. $0 v \beta \beta$ with CUORE}

Double beta decay is a second order weak interaction, only directly observable in even-even nuclei, for which the standard beta decay is suppressed or forbidden. The observation of these decays, in particular for neutrinoless double beta decay $(0 v \beta \beta)$ allows searching for possible deviations from the standard model. $0 v \beta \beta$ consists, as the name suggests, in a double beta decay without emission of neutrinos $(Z, A) \rightarrow(Z+2, A)+2 e^{-}$. This decay is possible only if there is a violation of lepton number by two units and if neutrinos are Majorana particles, thus equal to their own antiparticles. Besides proving that the lepton number is not a symmetry of nature, the observation of this hypothetical decay would have several implications: the introduction of Majorana masses will result in an expansion of the standard model and at the same time will provide a reliable explanation for neutrinos mass generation and scale. The discovery of Majorana neutrinos will also help to constrain the absolute neutrino mass hierarchy and scale and will provide strong indications for baryogenesis via leptogenesis as a mechanism that leads to a matter-antimatter asymmetry in the universe.

There are a dozen candidate isotopes for $0 v \beta \beta$ decay, but only a handful of them are used in experiments because of their high Q-value, isotopic abundance or their availability in nature. The isotope of interest for the CUORE experiment, whose results we will discuss in the following, is ${ }^{130} \mathrm{Te}$. This isotope has been chosen for its high isotopic abundance (34.17\%), its high Q-value of $2527.515 \pm 0.013 \mathrm{keV}$ [1] above most of the $\beta$-decay $/ \gamma$-ray background due to environmental radioactivity, and the reproducible growth of the crystals. Such high isotopic abundance allows using as detector $\mathrm{TeO}_{2}$ crystals produced with natural Te without having to resort to isotopic enrichment.

The signature for $0 v \beta \beta$ is a small peak at the end-point of the ${ }^{130} \mathrm{Te} 2 v \beta \beta$ spectrum. It is therefore crucial to have a low background index, thus the minimum possible number of events in the region of interest, otherwise the background could overrun a possible $0 v \beta \beta$ peak. The CUORE design includes passive background mitigation as well as a strict selection in the detector components to achieve a low background index. A detailed discussion on CUORE background budget can be found in [2] where it is shown how the projected background budget is within the CUORE goal of 0.01 counts $/ \mathrm{keV} / \mathrm{kg} / \mathrm{yr}$. Even in the hypothesis of a negligible background due to radioactive contamination in the detector materials, the two neutrino beta spectrum from ${ }^{130} \mathrm{Te}$ represents an irreducible source of events that leeks into the region of interest. An experiment with a good energy resolution can, however, be able to clearly distinguish the contribution from the two neutrino spectrum and that of $0 v \beta \beta$. We can control the energy resolution by maintaining the detector operation conditions as stable as possible. In the following, we discuss a new noise reduction technique that allows compensating for vibrational noise caused by the cryocoolers. The goal concerning energy resolution is $5 \mathrm{keV}$ FWHM, already proven to be possible by the pilot experiment CUORE-0 [3].

\section{The CUORE detector}

The Cryogenic Underground Observatory for Rare Events (CUORE) is a bolometric experiment operated in the underground national laboratories of Gran Sasso (LNGS), in central Italy. The underground location naturally protects the experiment from cosmic rays providing a shield 


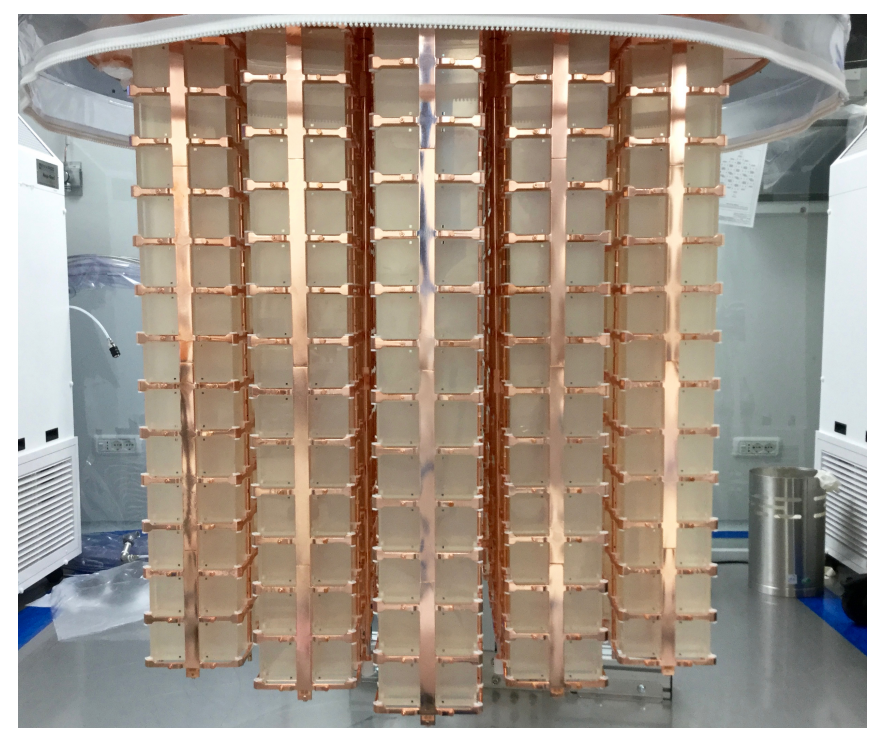

Figure 1: Picture of the CUORE towers during the detector installation: this was the only time in which the towers where exposed to air.

of $3600 \mathrm{~m}$ water equivalent. At the same time, the experiment is provided with passive barriers to reduce the background due to environmental radioactivity. The most external shield consists of polyethylene panels to moderate neutrons followed by panels of boric acid designed to capture the incoming thermal neutrons. The last outer shield, built with lead, absorbs $\gamma$-rays due to the laboratory natural radioactivity. The detector is hosted in a cryogen-free cryostat suspended on a structure designed to decouple the experiment from the rest of the laboratory and isolate it from vibrations, especially those due to earthquakes. The cryostat allows for cooling down about 15 tons of material below $4 \mathrm{~K}$ and in the inner vessel, where the $\mathrm{TeO}_{2}$ crystals are, it can reach temperatures below $10 \mathrm{mK}$. Inside the cryostat, there is an additional $6 \mathrm{~cm}$ thick layer of lead shielding. Given its proximity to the detector, this time archeological Roman lead has been chosen instead of modern lead. The archeological Roman lead, dated I century B.C. and found on a Roman shipwreck close to Sardinia coast, has the characteristic of being depleted in ${ }^{210} \mathrm{~Pb}$, essential if you want to keep the radioactive contamination as low as possible.

In the most inner part of the cryostat are hosted $988 \mathrm{TeO}_{2}$ crystals cubes of $5 \mathrm{~cm}$ per side, arranged in 19 towers, each one containing 52 crystals. Figure 1 shows a picture of the detector towers at the end of the installation inside the cryostat. In CUORE the $\mathrm{TeO}_{2}$ crystals are both absorbers and source of the decay of interest. We can measure the small increase in temperature caused by particle interaction by mean of a neutron transmutation doped Germanium thermistors (NTD) [4]. The temperature of operation for the data presented here is $15 \mathrm{mK}$; however, we have foreseen to continue the data taking at a slightly lower temperature.

\section{Detector optimization}

The CUORE cryostat is cooled down to about $10 \mathrm{mK}$ thanks an ${ }^{3} \mathrm{He} /{ }^{4} \mathrm{He}$ dilution refrigerator. Dilutions, however, requires a $4 \mathrm{~K}$ environment, provided by four mechanical cryocoolers, the 
Pulse Tubes (PTs). With respect to the use of a liquid He bath to reach the $4 \mathrm{~K}$ stage, PTs provide numerous advantages, in particular, the increased duty cycle, scalability and dismissal of any manual intervention on the cryostat during data taking. The use of PTs has, on the other hand, a limiting factor: the cryocoolers cause mechanical vibrations due to the rotation of the motor head rotary valve and the pressure waves of the compressor necessary for their operation. Typical frequencies induced by the PTs are $1.4 \mathrm{~Hz}$ and its harmonics, which fall within the characteristic bolometer signal frequency band. Despite the implementation of passive vibration dumping and attenuation techniques, these frequencies often transmit vibrations to the detectors through cables and support mechanisms. Moreover, since the frequencies induced by the PTs are not precisely $1.4 \mathrm{~Hz}$, the CUORE detector suffers vibrations that are not constant but modulate in time with an interference pattern that depends on the position of the single crystal in the cryostat with respect of the four PTs. The presence of nonconstant sources of noise degrades the signal to noise ratio, compromising the sensitivity of the experiment. During the firsts months of operation, an innovative technique of noise cancellation for the PTs induced vibrations has been developed [5]. The first step for the noise cancellation of the PT induced noise is to replace the embedded controllers of the rotary valves of the PTs, not optimized for low noise, with an LNX Linear Series low-noise drive from Precision Motion Control that from now on we will call Linear Drive (LD). The LDs are micro-stepping precision motors whose effect is the attenuation of the vibrations thanks to an increased number of steps in the rotation, thus a smoother movement of the mechanical parts. The LDs allow also controlling the rotation of the PT rotary valve by small accelerations or decelerations.

The second step for the PT induced noise cancellation consisted in the development of custom software that allows correcting the velocity of the rotary valves continuously and stabilize in time the characteristic noise of each bolometer.

The last step of the noise cancellation technique is the reduction of the overall noise amplitude due to PT induced frequencies. The procedure consists in studying the amplitude of the frequency peaks in the noise power spectrum of the signal from each bolometer. We monitor the frequencies that most influence the signal to noise ratio in the detector while scanning the available phase space of the phase differences in the beating of the four PTs. We finally choose the set of phase differences that minimizes the noise in the majority of the crystals. The chosen set of relative phases of the PT pressure oscillations causes coherent superposition of the vibrations transmitted to the entire cryogenic system, including the crystals, therefore partially canceling the noise. We can indeed observe the effects not only on the acquired data but also on the temperature of the cryostat whose result is more stable, as shown in Figure 2.

\section{First results}

In this last section, we will present the latest results of the $0 v \beta \beta$ decay analysis in CUORE [6] and the effect the noise optimization had on the energy resolution.

This analysis uses data collected during two periods: the first from May to June, which we will call DS1, and the second from August to September of 2017, which we will refer to as DS2. Each of these datasets has two calibrations associated: one at the beginning and one at the end of the 


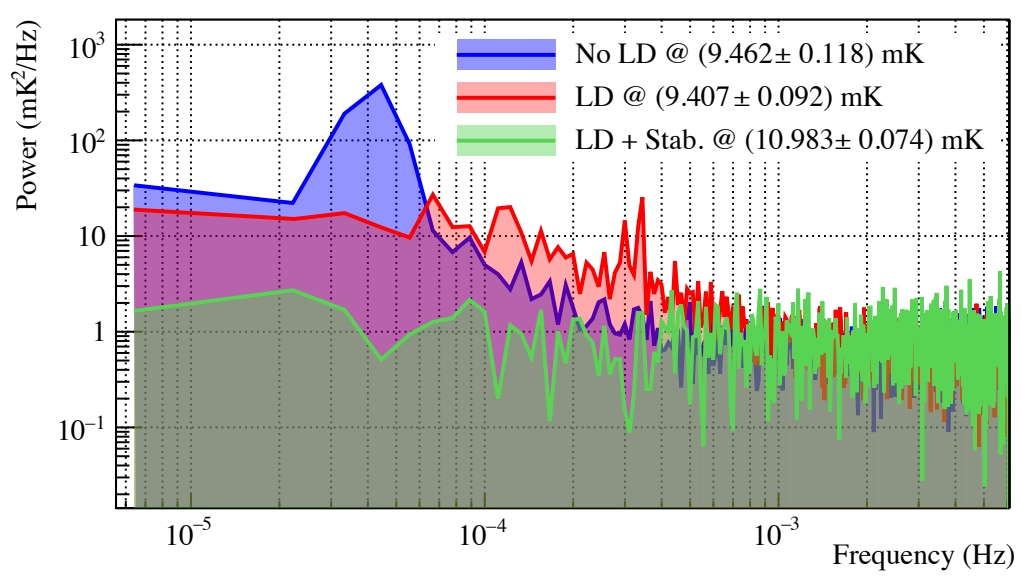

Figure 2: Effect of the different steps of the noise cancellation technique on the temperature of the coldest stage of the CUORE cryostat. The plot shows the noise power spectrum of the thermometer data acquired without any precaution (blue), after switching to LDs (red) and with both LDs and phase stabilization (green). Image from [5].

dataset. The calibration is performed using ${ }^{232} \mathrm{Th} \gamma$-ray sources [7] and its meant for both energy calibration and checking the stability of the detector response.

We dedicated most of the time between the first and the second dataset to optimizing the PTs noise cancellation system and improve the electrical grounding of the detector. In fact in the first dataset only the PTs phase stabilization to prevent the modulation of the noise was active, while for the second dataset the PTs phases where kept in a configuration that minimizes the noise on the detector.

Both datasets were analyzed together to guarantee uniformity in the data processing and the results. The amplitude of the pulses was estimated using waveform filtered with the optimal filter which suppresses frequencies typical of the noise waveform, enhancing the signal to noise ratio for particle-induced events. The pulse amplitude is then corrected for possible variation of the detector response due to small temperature fluctuations, exploiting heater induced events with a fixed power. The events are then calibrated using six $\gamma$ lines from the calibration runs and immediately blinded to proceed to the higher level analysis without introducing any bias.

The subsequent steps ensure the quality of the data: only events with a shape consistent with a true signal-like event and those events that are not in coincidence with any other, survive to the final $0 v \beta \beta$ fit. These cut are designed to remove pileup, noise spikes and events due to multiple scattering of $\gamma$ rays or muon induced showers. Then we determine the detector response to a monoenergetic event in the region from $2465 \mathrm{keV}$ to $2575 \mathrm{keV}$ using the high-statistics ${ }^{208} \mathrm{Tl} 2615$ $\mathrm{keV} \gamma$ line from calibration data and extrapolating the result to the region of interest. We model the ${ }^{208} \mathrm{Tl}$ line with a primary Gaussian component centered at $2615 \mathrm{keV}$ and two additional Gaussians, one on the sides of the central peak. We perform a channel and dataset dependent simultaneous fit to account for the differences in detector response of the CUORE channels. Finally, we use the detector response to model, in the region of interest, a peak for the $0 v \beta \beta$ decay and a peak for ${ }^{60} \mathrm{Co}$ 


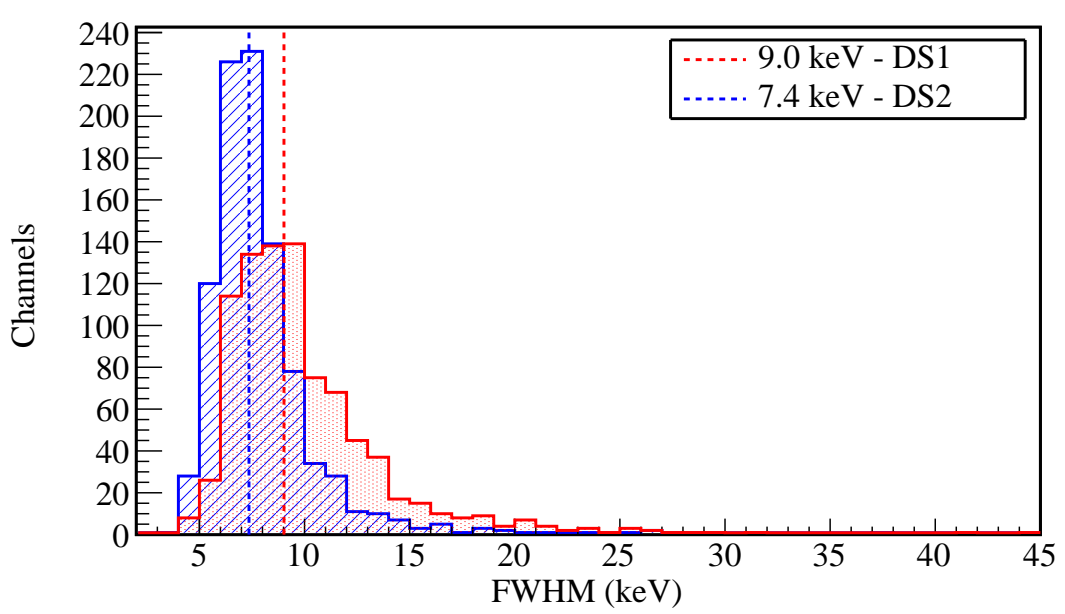

Figure 3: Distribution of energy resolution (FWHM) for the first (red) and second (blue) datasets. The vertical dotted lines indicate the weighted average of the corresponding distribution. From this plot it is clear that between the first and second dataset there has been a noticeable improvement in the energy resolution.

coincident $\gamma$ rays, on top of a flat background. From the simultaneous fit of the region of interest we extract the best fit for the $0 v \beta \beta$ decay event rate and from it we extract a lower limit for the half-life of ${ }^{130} \mathrm{Te} 0 v \beta \beta$ decay, which is at $90 \%$ confidence limits $T_{1 / 2}^{0 v}>1.3 \times 10^{25} \mathrm{yr}$, including systematics.

In addition to the detector response, we can use the fit on the ${ }^{208} \mathrm{Tl}$ line to obtain the energy resolution of each bolometer. We report the distribution of the energy resolution (FWHM) for both datasets in Figure 3. We conclude stressing that the improvement of the second dataset compared to the first one is probably due to the noise reduction campaign and it opens up the possibility of further improvements of the overall energy resolution if we keep the vibrational noise under control.

\section{Acknowledgments}

This work was supported by the Istituto Nazionale di Fisica Nucleare (INFN); the National Science Foundation under Grant Nos. NSF-PHY-0605119, NSF-PHY-0500337, NSF-PHY-0855314, NSF-PHY-0902171, NSF-PHY-0969852, NSF-PHY-1307204, and NSF-PHY-1404205; the Alfred P. Sloan Foundation; and Yale University. This material is also based upon work supported by the US Department of Energy (DOE) Office of Science under Contract Nos. DE-AC02-05CH11231 and DE-AC52-07NA27344; and by the DOE Office of Science, Office of Nuclear Physics under Contract Nos. DE-FG02-08ER41551, DE-FG03-00ER41138, DE-SC0011091, and DE-SC0012654. This research used resources of the National Energy Research Scientific Computing Center (NERSC). 


\section{References}

[1] S.Rahaman et al. Phys. Lett. B vol. 704 4, 412-416 (2011).

[2] C. Alduino et al. (CUORE Collboration), Eur. Phys. J. C vol. 77 , 543 (2017).

[3] C. Alduino et al. (CUORE Collboration), Phys. Rev. C vol. 93 , 045503 (2016).

[4] J. Beeman et al. Phys. Rev. B vol. 41 6, 3761-3768 (1990).

[5] A. D'Addabbo et al., Cryogenics vol. 93 , 56-65 (2018).

[6] C. Alduino et al., (CUORE Collboration), Phys. Rev. Lett. vol. 120 no. 13, 132501 (2018).

[7] J.S. Cushman et al., Nucl. Instrum. Methods A vol. 844 , 32-44 (2017).

[8] D.R. Artusa et al. (CUORE Collaboration), Adv. High Energy Phys. , 879871 (2015).

[9] K. Alfonso et al. (CUORE Collboration), Phys. Rev. Lett. vol. 115 , 102502 (2015). 